\title{
Test facility management as per good laboratory practice
}

\section{Editorial}

Test Facility Management?" [TFM] means the person(s) who has the authority and formal responsibility for the organization and functioning of the test facility according to the principles of OECDGood Laboratory Practice. ${ }^{1}$ Test Facility can be managed by one or more persons at one or more locations. Test Facility Management has overall responsibility of facility management, study personnel, and quality assurance programme. It is the responsibility of the testing facility management to ensure that clear lines of communication exist and that these lines of communication are clearly documented. For any non clinical study, scientist who is qualified and experienced in conducting study has to be appointed as study director. There should be independent quality assurance unit who are not part of conducting the GLP study. They are entirely separate from and independent of the personnel engaged in the direction and conduct of that study. In short Test Facility Management is defined as each individual. Responsible for the supervision of a nonclinical laboratory study shall have education, training, and experience, or combination thereof, to enable that individual to perform the assigned functions. The organizational responsibility, first and foremost is that to ensure the facility operates in compliance with the OECD principles of good laboratory practice

\section{Test facility management as per good laboratory practice}

i. Organizational chart: describes how the facility functions

ii. Master Schedule: ensure that all studies, contracted or inhouse, are included

iii. CV and JD: A record of the qualifications, training, experience, and job description for each individual should be maintained.

iv. SOP's: Appropriate and technically valid SOPs should be established and followed, and all original and revised SOPs should be approved

v. Quality Assurance Unit: designated and adequately trained personnel should be established. The quality assurance responsibility should be performed in compliance to the principles of good laboratory practice.

vi. Study director: A study director with the appropriate qualifications, training, and experience should be designated for each study before the study is initiated. A study director should be replaced, if necessary, according to established procedures and should be properly documented.

vii. Principal investigator: For a multi-site study, a principal investigator should be designated. The principal investigator should be appropriately trained, qualified, and experienced to supervise the delegated phase of the study. A principal investigator should be replaced, if necessary, according to established procedures and should be properly documented.
Volume 3 Issue 4 - 2017

\author{
Pradeep Deshmukh \\ PRADO-Preclinical Research and Development Organisation, \\ India
}

Correspondence: Pradeep Deshmukh, PRADO-Preclinical Research and Development Organisation, India,

Email priyankabhaskaran1993@gmail.com

Received: August 16,2017| Published: August 17, 2017

viii. Study plan/Protocol: The study director has approved the study plan and has made the approved study plan available to the quality assurance personnel

ix. Archives: An individual should be designated who is responsible for the management of the archives.

x. Suppliers: The testing facility supplies should meet the requirements appropriate to their use in a study.

xi. Communication: For a multi-site study, clear lines of communication should exist between the study director, principal investigator, the quality assurance program(s), and study personnel

xii. Characterisation of test and reference items: Test and reference items should be appropriately characterized.

xiii. Computerized systems: Procedures should be in place to ensure that computerized systems are suitable for their intended purpose and are validated, operated, and maintained in accordance with the principles of good laboratory practice.

\section{Test facility management and documents}

Test facility management should keep the following documents ready for GLP inspection:
i. Floor plans
ii. organization charts
iii. CVs of personnel involved in a study

iv. Master Schedule: List of ongoing and completed studies with information on the type of study, initiation and completion dates, test system, method of application of test substance, and name of the study director

v. Staff health surveillance policies

vi. Staff job descriptions and staff training programs and records

vii. List of Standard Operating Procedures

viii. Specific SOPs related non clinical toxicology studies 
ix. List of the study directors

x. Job descriptions and staff training records

\section{Summary}

In summary, there are important principles of good laboratory practices. These are resources, characterization, rules, results, and quality assurance. Test Facility Management has a significant responsibility in the management of non clinical toxicology studies conducted in the test facility. Adequate should be in place to assure the integrity and validity of the study and its data support product safety. To assure data integrity, there are many controls required including equipment calibration and maintenance, training of study personnel, careful preparation and review of study plans and testing procedures, validation of test methods, qualification of materials, handling and care of animals-test systems, validation of computer systems used data analysis, monitoring of studies by the quality assurance unit, and much more. ${ }^{2}$ The oversight and control of all aspects of GLP studies conducted by the testing facility are the responsibility of Test Facility Management.

\section{Acknowledgements}

None.

\section{Conflict of interest}

The author declares no conflict of interest.

\section{References}

1. Handbook-Good Laboratory Practice (GLP). India: UNDP/World Bank/WHO.

2. Good Laboratory Practice. (GLP)-Training Manual. 2nd ed. India: WHO; 2008. 